\title{
The Nucleon-Nucleon Interaction
}

\author{
J. Haidenbauer \\ Forschungszentrum Jülich, Institut für Kernphysik, D-52425 Jülich, Germany
}

Received on 11 September, 2003

\begin{abstract}
Recent developements in our understanding of the fundamental nucleon-nucleon interaction are reviewed. Among the topics considered are (1) the so-called high-precision nucleon-nucleon potentials that emerged in the mid 1990s, (2) progress in the microscopic description of the intermediate-range part of the nucleon-nucleon interaction in terms of correlated pion-pion and rho-pion exchange and (3) efforts towards a quantitative descripton of the nucleon-nucleon interaction utilizing methods based on effective field theory.
\end{abstract}

\section{Introduction}

In my talk I want to give a brief overview of recent developements in our understanding of the fundamental nucleonnucleon $(N N)$ interaction. Naturally, such a review will be biased and it focusses on aspects and topics that I consider to be important.

During the 1990s several groups have constructed socalled high-precision, charge-dependent $N N$ potentials. These models provide a rather accurate description of the empirical $N N$ phase shifts, however, they are essentially phenomenological. I review these models and discuss their merits as well as their drawbacks in Sec. 2.

Reseach in an entirely different direction was conducted by the Jülich group. Here the main aim was a microscopic understanding of the $N N$ interaction at intermediate and short ranges. In this context the Jülich group developed a microscopic model for correlated $\pi \pi$ as well as $K \bar{K}$ exchange between baryons that allows to replace the exchange of the fictitious (scalar-isoscalar) $\sigma$ meson, which is present in basically all one-boson exchange models of the $N N$ interaction. Furthermore the contributions of correlated $\pi \rho$ exchange were considered. I present corresponding results in Sec. 3.

Motivated by successful applications of chiral perturbation theory in the $\pi \pi$ and $\pi N$ sector, Weinberg proposed in 1990 to extend the formalism to the $N N$ interaction. His suggestion was picked up by the Texas-Seattle group in the mid 1990s and then perfected by groups in São Paulo, Jülich and Idaho. I report on the present status of those investigations in Sec. 4.

Possible goals for future research are discussed in Sec. 5. This concerns specifically an extension of the interaction models to higher energies, i.e. up to laboratory energies of $2-3 \mathrm{GeV}$.

\section{High precision $N N$ potentials}

In the 1990s several so-called high precision $N N$ potentials were developed $[1,2,3]$. Starting point of this de- velopment was a new partial wave analysis for the energy region $T_{l a b} \leq 300 \mathrm{MeV}$ carried out by the Nijmegen group [4]. The main new ideas in this analysis were that (i) the data base was "pruned", i.e. data with either an improbably high $\chi^{2}$ or with an improbably low $\chi^{2}$ were eliminated and (ii) the analysis was constrained by using a potential (one pion exchange plus part of the two-pion exchange) for the long-range part of the $N N$ interaction. This potential, supplemented with a boundary condition that parametrizes the short-range part of the $N N$ interaction, was inserted in the Schrödinger equation and the parameters of the latter were adjusted in order to achieve an optimal description of the $N N$ data. In this way a partial wave representation with a $\chi^{2} /$ datum of around 1 could be achieved [4].

High precision $N N$ potentials aim at a description of the $N N$ phase shifts with the same quality, i.e. with $\chi^{2} /$ datum $\approx 1$. Thus, they provide an accurate and reliable representation of our empirical information on the $N N$ interaction. For comparison, conventional $N N$ potentials like the Paris, (full) Bonn, or Argonne V14 models have typically $\chi^{2} /$ datum $\approx 2$.

What are the merits of high precision potentials? Since their results are basically equivalent to the phase shift analysis they provide a perfect description of the available data on the $N N$ interaction. (Indeed even the often small effects from charge dependence are taken into account.) Thus, the application of such potentials in microscopic calculations of few- and many-body systems $\left({ }^{3} \mathrm{H}, N-d\right.$ scattering, ${ }^{4} \mathrm{He}$, ..., nuclear matter) guarantees that the $N N$ properties have been taken into account reliably so that remaining discrepancies with data should then be due to other sources such as three-body forces, relativistic effects, etc. On the other hand, one must admit that those high precision $N N$ potentials tell us very little about the $N N$ dynamics itself. Besides the one-pion exchange they are basically built on phenomenological ingredients. Strictly speaking this is true only for the Argonne V18 model [2] and the Readlike potential (Read93) of the Nijmegen group [1]. However, even in the OBE models (CD-Bonn [3], NijmI, NijmII [1]) some coupling constants and/or form factors are adjusted individually for each partial wave in order to optimize the results. 


\section{Role of meson-meson correlations in the $N N$ interaction}

It is known for a long time that meson-meson correlations play an important role in baryon-baryon interactions. For example, the intermediate-range attraction that is required for a description of the $N N$ interaction is commonly believed to be due to correlated $\pi \pi$-exchange processes in the $J^{P}=0^{+}$state of the $t$ channel. However, in essentially all potentials this contribution is parametrized by the use of a fictitious scalar-isoscalar boson, the $\sigma$. The Jülich group has developed a microscopic model for correlated $\pi \pi$ as well as $K \bar{K}$ exchange between baryons that allows to replace the sharp mass $\sigma$ (and $\rho$ ) exchange. Corresponding results can be found in Ref. [5].

There are still some other open questions, e.g., the true nature of the $N N$ repulsion. In meson-exchange models like the Bonn potentials [6] the repulsion is provided only by $\omega$ meson exchange. As a consequence an $\omega$ coupling constant of $g_{N N \omega}^{2} / 4 \pi \approx 20$ is required for achieving a satisfactory description of $N N$ phase shifts - which is much larger than the value of $g_{N N \omega}^{2} / 4 \pi \approx 9$ that follows from $\mathrm{SU}(3)$ symmetry. This is rather surprising because we know from other studies, e.g. of the hyperon-nucleon interaction $[7,8]$, that $\mathrm{SU}(3)$ symmetry is roughly fulfilled. Another open issue is the $\pi N N$ form factor which is an expression of the extended structure of hadrons and is usually characterized in a monopole parametrization by a cutoff mass $\Lambda_{\pi N N}$. Within the (full) Bonn potential [6] a value of $\Lambda_{\pi N N}=1.3 \mathrm{GeV}$ is required, whereas informations from other sources $[9,10]$ favour considerably lower values, $\Lambda_{\pi N N} \approx 0.8 \mathrm{GeV}$. Studies by the Jülich group made clear that both these problems have to do with the role of correlated $\pi \rho$ exchange.
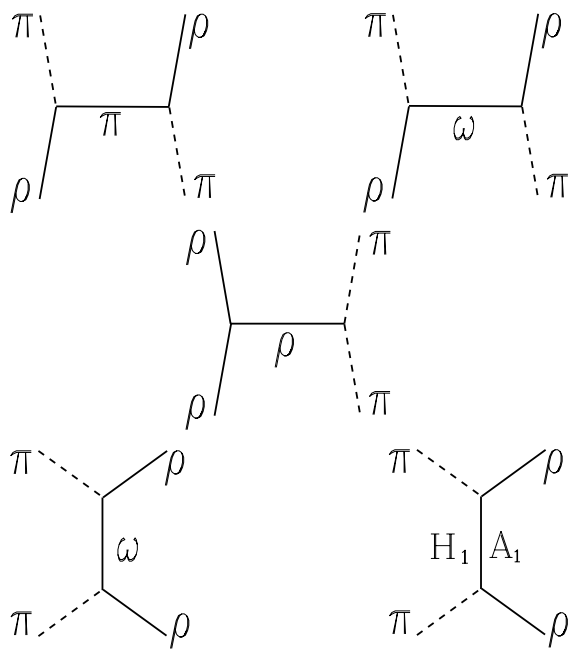

Figure 1. Born terms included in the model of Ref. [11] for the $\pi \rho$ interaction.

The importance of $\rho$-exchange for the dynamics of the $N N$ system derives from the following fact: It provides a sizable intermediate range tensor force, which has opposite sign to the tensor force generated by one-pion exchange.
Thus there is a strong cancellation, over a relatively broad range of energies and distances, between $\pi$ and $\rho$ exchange in the tensor channel. A similar cancellation occurs between $\mathrm{K}$ and $\mathrm{K}^{*}$ exchange, e.g. in the hyperon-nucleon interaction. Therefore, in the $N N$ (and in the baryon-baryon system in general) it is strongly suggested to always group $\pi$ and $\rho$ (as well as $\mathrm{K}$ and $\mathrm{K}^{*}$ ) together in order to reach sufficient convergence in the expansion of the irreducible kernel (potential).

In fact this procedure has been an essential guideline when constructing the Bonn potential [6]. Unfortunately it was not followed to a sufficient degree: Whereas, in second order diagrams (cf. Ref. [6]) $\pi \pi$ as well as $\pi \rho$ exchange has been included for uncorrelated processes (with $N$ and $\Delta$ intermediate states) this has not been done for correlated processes: correlated $2 \pi$-exchange processes have been effectively included (in terms of sharp mass $\sigma^{\prime}$ and $\rho$ exchange) but correlated $\pi \rho$ processes have been left out. The reason is quite simple: The evaluation of this missing piece is technically quite complicated, much more involved (due to the spin of the $\rho$ ) compared to correlated $2 \pi$-exchange. More importantly, a dynamical model for the interaction between a $\pi$ and a $\rho$ meson was not available at that time.

Such a potential model has been constructed by the Jülich group in the meantime [11], with driving terms shown in Fig. 1. Open parameters have been adjusted to empirical pole parameters of the $a_{1}, h_{1}$, and $\omega$ meson. This $\pi \rho$ T-matrix is now inserted into the correlated $\pi \rho$ exchange diagram of Fig. 2. The evaluation proceeds via the same dispersion-theoretical treatment as used for the $\pi \pi$ case in Ref. [5].

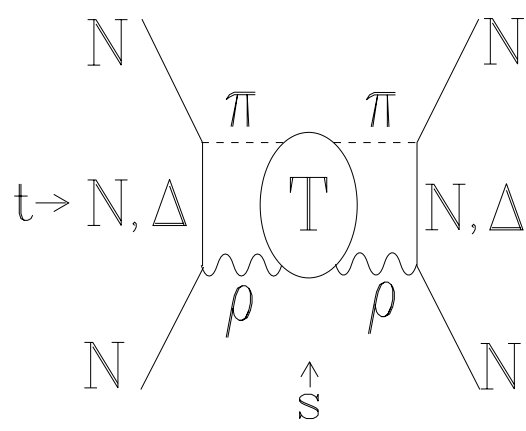

Figure 2. Correlated $\pi \rho$ exchange.

Like for correlated $\pi \pi$ exchange the result can be represented as an integral over various spectral functions in the different channels $J^{P}\left(I^{G}\right)=0^{-}\left(1^{-}\right)(" \pi "), 1^{-}\left(0^{-}\right)$(" $\left.\omega^{\prime \prime}\right)$, $1^{+}\left(1^{-}\right)$("a $\left.a_{1}^{\prime}\right), 1^{+}\left(0^{-}\right)$(" $\left.h_{1} "\right)$ which we have considered [12]. Thus we have for the pionic channel:

$$
V_{N, N ; N, N}^{\left(0^{-}\right)}(t) \sim \int_{\left(m_{\pi}+m_{\rho}\right)^{2}}^{\infty} d t^{\prime} \frac{\rho^{\left(0^{-}\right)}\left(t^{\prime}\right)}{t^{\prime}-t} .
$$

The spectral function $\rho^{\left(0^{-}\right)}$characterizes both the strength and the range of the interaction and it is shown in Fig. 3. 
Obviously correlated $\pi \rho$ exchange in the $0^{-}\left(1^{-}\right)$channel provides a sizable contribution, with a peak around $1.1 \mathrm{GeV}$, somewhat smaller than the mass $(1.2 \mathrm{GeV})$ of the phenomenological $\pi^{\prime}$ introduced in a model study of the $N N$ interaction that tried to accommodate a soft $\pi N N$ form factor [13].

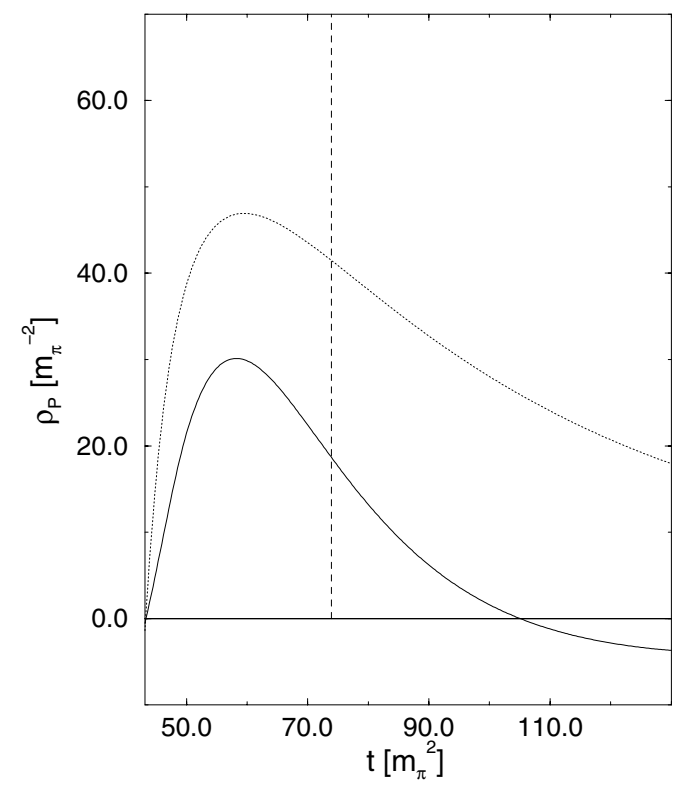

Figure 3. The spectral function in the pionic channel $\rho_{P}^{\pi}(t)$. The dotted line shows the uncorrelated part whereas the solid line represents the correlated contribution. The vertical dashed line represents sharp mass $\pi^{\prime}$ exchange used in Ref. [13].
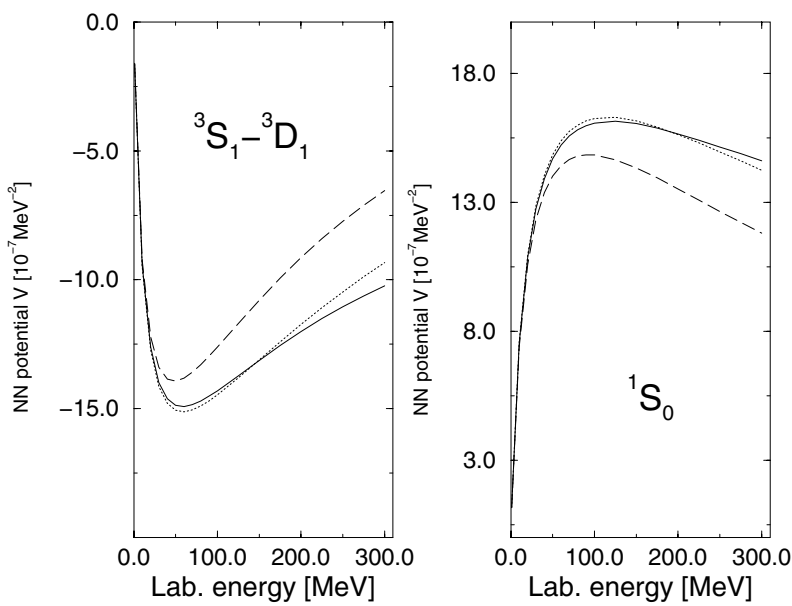

Figure 4. On-shell $N N$ potential $V_{N N}$ as function of the nucleon lab energy for the ${ }^{3} D_{1}-{ }^{3} S_{1}$ transition (lefthand side) and in the ${ }^{1} S_{0}$ state (righthand side). The dotted line denotes the one-pion-exchange potential as used in the Bonn potential $\left(g_{\pi N N}^{2} / 4 \pi=14.4, \Lambda_{\pi N N}=1.3 \mathrm{GeV}\right)$. For the dashed line, $\Lambda_{\pi N N}=1.0 \mathrm{GeV}$ was used. The solid line results if correlated $\pi \rho$ exchange in the pionic channel is added to the dashed line.

Indeed as shown in Fig. 4, the resulting interaction due to correlated $\pi \rho$ exchange in the pionic channel is able to counterbalance the substantial suppression induced in the one pion-exchange potential when going from a cutoff mass $\Lambda_{N N \pi}$ of $1.3 \mathrm{GeV}$, phenomenologically required in the (full) Bonn potential, to a value of $1.0 \mathrm{GeV}$. For basic theoretical reasons such a reduction is highly welcome, since various information from other sources point to a rather soft $\pi N N$ form factor characterized by $\Lambda_{N N \pi} \cong 0.8 \mathrm{GeV}[9,10]$. Indeed this value can be reached if correlated $\pi \sigma$ exchange is included too [14], which is also missing in the Bonn potential. (As usual, $\sigma$ stands for a low mass correlated $\pi \pi$ pair in the $0^{+}$channel). Thus it appears that in the Bonn potential one pion-exchange together with a hard form factor is an effective description of 'true' one-pion exchange (with a soft form factor) plus correlated $\pi \rho$ (and $\pi \sigma$ ) exchange in the pionic channel.

In the $\omega$ channel, the exchange of a correlated $\pi \rho$ pair also provides a sizable contribution to the $N N$ interaction. Here we have included the genuine $\omega$ meson explicitly and replaced the (effective) $\omega$ exchange in the Bonn potential by the resulting correlated $\pi \rho$ potential, which can be decomposed into a pole and a non-pole piece. The former provides a microscopic model for 'true' $\omega$ exchange leading to a renormalized $\omega N N$ coupling constant at the pole, $g_{N N \omega}^{2} / 4 \pi=11.0$, which is about a factor of 2 smaller than the effective value of 20 used in the Bonn potential. Thus 'true' correlated $\pi \rho$ exchange, i.e. the non-pole piece, provides almost half of the empirical repulsion needed in the $N N$ interaction. It can be roughly parametrized by sharpmass $\omega^{\prime}$-exchange with $g_{N N \omega}^{2} / 4 \pi=8.5, f_{N N \omega} / g_{N N \omega}=0.4$ and $m_{\omega^{\prime}}=1120 \mathrm{MeV}$ [12].

The new reduced coupling constant (11.0) is still about a factor of 1.5 larger than provided by customary SU(3) estimates, which use $g_{N N \omega}^{2}=9 g_{N N \rho}^{2}$. Thus with $g_{N N \rho}^{2} / 4 \pi=$ 0.84 as used in the full Bonn model [6] we have $g_{N N \omega}^{2} / 4 \pi=$ 7.5. Note however, that the above relation between $\omega$ and $\rho$ coupling constants is based, apart from ideal mixing, on the assumption of vanishing $\phi N N$ coupling. For $g_{N N \phi}$ unequal to zero the above relation goes into

$$
g_{N N \omega}=3 g_{N N \rho}-\sqrt{2} g_{N N \phi} .
$$

If we take $g_{N N \phi}=-0.5$, (which amounts to a rather small deviation from zero) we have $g_{N N \omega}^{2} \approx 9$, which already comes close to our results. Such a value for the $\phi N N$ coupling to the nucleon and the negative sign is quite conceivable from the $\phi$ coupling to the nucleon via the $\Lambda \bar{\Lambda}$ continuum [15].

\section{Approaches based on effective field theory}

The application of methods of effective field theories to the $N N$ interaction will be discussed thoroughly in the contribution of M. Robilota [16]. Therefore, I shall be rather brief here. Chiral perturbation theory has been rather successfully used in studies of the $\pi \pi$ and $\pi N$ systems at low energies. Thus, it is natural to apply this approach to the $N N$ system 
TABLE 1: $\chi^{2} /$ datum for the reproduction of the $1999 n p$ database [3] below $290 \mathrm{MeV}$ by various $n p$ potentials.

\begin{tabular}{cccccc}
\hline \hline Bin (MeV) & \# of data & $\mathrm{N}^{3} \mathrm{LO}^{a}$ & $\mathrm{NNLO}^{b}$ & $\mathrm{NLO}^{b}$ & ${\mathrm{AV} 18^{c}}^{c}$ \\
\hline $0-100$ & 1058 & 1.06 & 1.71 & 5.20 & 0.95 \\
$100-190$ & 501 & 1.08 & 12.9 & 49.3 & 1.10 \\
$190-290$ & 843 & 1.15 & 19.2 & 68.3 & 1.11 \\
\hline $0-290$ & 2402 & 1.10 & 10.1 & 36.2 & 1.04 \\
\hline \hline
\end{tabular}

${ }^{a}$ Ref. [28], ${ }^{b}$ Ref. [25], ${ }^{c}$ Ref. [2].

as well, specifically because the pion plays also an important role in the $N N$ interaction. However, this cannot be done immediately. The $N N$ interaction at low energies is characterized by large scattering lengths and even shallow bound states, i.e. features which are intrinsically non-perturbative. Because of that Weinberg [17] suggested that one should expand the kernel (the "potential") rather than the scattering amplitude and then insert this potential into a scattering (Lippmann-Schwinger) equation. In this case a systematic expansion of the nuclear amplitude in terms of $\left(Q / \Lambda_{\chi}\right)^{\nu}$ is possible, where $Q$ is the external nucleon momentum or the pion mass, $\Lambda_{\chi}$ is the chiral symmetry breaking scale $(\approx 1$ $\mathrm{GeV})$ and $\nu \geq 0$ specifies the order of the expansion. This idea was first applied to the $N N$ system by van Kolck and Ordóñez $[18,19]$ and lateron refined by collaborations in São Paulo [20, 21], Munich [22, 23], Jülich [24, 25, 26] and Idaho [27, 28].

Table 1, taken from Ref. [28], summarizes the present state-of-art of calculations in this direction. Evidently, results for the $N N$ phase shifts with a quality comparable to those of high precision $N N$ interactions can be obtained if one goes to sufficiently high order which is $\mathrm{N}^{3} \mathrm{LO}$ (nextto-next-to-next-to-leading-order) and corresponds to $\nu \leq 4$ in the momentum expansion mentioned above.

\section{Future directions}

Over the last decade or so the $N N$ data base in the energy region of $500 \mathrm{MeV} \leq T_{l a b} \leq 2.5 \mathrm{GeV}$ has been significantly improved (though essentially for $p p$ only). This developement was driven primarily by experimentalists at the SATURNE II accelerator at Saclay [29] and by the EDDA collaboration at the COSY facility in Jülich [30], and led to rather accurate data not only on cross sections but also on spin-dependent observables. As a consequence, a more refined phase shift analysis has become feasible up to such high energies [31]. However, this progress was not matched on the theoretical side. Basically all $N N$ models I mentioned earlier are only valid up to around $T_{l a b}=300 \mathrm{MeV}$, i.e. up to the pion-production threshold. These models include no inelastic channels and, therefore, cannot be confronted with data at higher energies. There are, however, a few models in the literature which take into account the coupling of the $N N$ system to the $N \Delta$ and $\Delta \Delta$ channels, e.g. the ones in Refs. [32, 33]. These models are, in general, able to describe the $N N$ phase shifts and inelasticities up to around $T_{l a b} \approx 1.2 \mathrm{GeV}$ - though more qualitatively than quantitatively. But for even higher energies the models are too repulsive, yielding large negative phase shifts that are not in agreement with the empirical results, and, moreover, predict too small inelasticity parameters. It is clear that new dynamical ingredients are needed. One of the obvious modifications would be the inclusion of further inelastic channels in those models. This concerns specifically the coupling to the $N N^{*}(1440)$ and $N N^{*}(1535)$ channels which have their thresholds at $T_{l a b}=1140 \mathrm{MeV}$ and $1380 \mathrm{MeV}$, respectively, and therefore should be important in the energy range above $1.2 \mathrm{GeV}$. Furthermore, since at such high energies one is getting more and more sensitive to the details of the short-range part of the $N N$ interaction, improvements should be made there as well. First of all one should include exchange contributions of mesons with higher masses, and specifically those of the axial vector mesons $a_{1}(1260)$, $b_{1}(1235), h_{1}(1170)$, and $f_{1}(1285)$. Also, it is conceivable that a more refined treatment of the vertex form factors is required. So far these are simply parametrized by monopole and/or dipole forms. Finally, one should explore the possibility of an explicit inclusion of quark-gluon degrees of freedom into meson-exchange models [34, 35]. Investigations along these lines are presently under way in Jülich.

\section{Summary}

I have shown you that the $N N$ interaction in the energy region $T_{l a b} \leq 300 \mathrm{MeV}$ is now rather well under control. Good quantitative results have been achieved with phenomenological potentials, with conventional mesonexchange models, but also with interaction models derived by employing methods of effective field theories (chiral perturbation theory).

On the other hand, the understanding of the $N N$ interaction in the energy region of $500 \mathrm{MeV} \leq T_{l a b} \leq 2.5 \mathrm{GeV}$, say, is still a challenge. The data base at these higher energies has been significantly improved over the last 10 years or so (though for $p p$ only), not least due to the efforts of the EDDA collaboration at the COSY facility in Jülich, and a more refined phase shift analysis has become feasible. Therefore, the way is prepared for extending models of the $N N$ interaction to such energies. Besides technical aspects this will allow to address several interesting general questions like: Is the meson-exchange picture still appropriate at such energies? Do we need/see explicit quark-gluon degrees of freedom? Are other approaches such as Regge-type models or even $\mathrm{pQCD}$ already more promising? 


\section{References}

[1] V. G. J. Stoks, et al., Phys. Rev. C 49, 2950 (1994).

[2] R. B. Wiringa et al., Phys. Rev. C 51, 38 (1995).

[3] R. Machleidt, Phys. Rev. C 63, 024001 (2001).

[4] V. G. J. Stoks, et al., Phys. Rev. C 48, 792 (1993).

[5] H.-C. Kim, J. W. Durso, and K. Holinde, Phys. Rev. C 49, 2355 (1994); A. Reuber, K. Holinde, H.-C. Kim, Nucl. Phys. A608, 243 (1996).

[6] R. Machleidt, K. Holinde, and Ch. Elster, Phys. Rep. 149, 1 (1987).

[7] B. Holzenkamp et al., Nucl. Phys. A500, 485 (1989); A. Reuber et al., Nucl. Phys. A570, 543 (1994)

[8] J. Haidenbauer et al., AIP Conf. Proc. no. 603 (2001), pp. 421; nucl-th/0108062.

[9] S.A. Coon, and M.D. Scadron, Phys. Rev. D42, 2256 (1990).

[10] K.-F. Liu et al., Phys. Rev. Lett. 74, 2172 (1995).

[11] G. Janssen, K. Holinde, and J. Speth, Phys. Rev. C 49, 2763 (1994).

[12] G. Janssen, K. Holinde, and J. Speth, Phys. Rev. C 54, 2218 (1996).

[13] J. Haidenbauer, K. Holinde, and A. W. Thomas, Phys. Rev. C49, 2331 (1994).

[14] R. Böckmann et al., Phys. Rev. C 60, 055212 (1999).

[15] U.-G. Meissner et al., Phys. Lett. B408, 381 (1997).

[16] M. Robilotta, contribution to this conference.

[17] S. Weinberg, Phys. Lett. B 251, 288 (1990); Nucl. Phys. B363, 3 (1991); Phys. Lett. B 295, 114 (1992).

[18] C. Ordóñez and U. van Kolck, Phys. Lett. B291, 459 (1992).

[19] C. Ordóñez, L. Ray, and U. van Kolck, Phys. Rev. Lett. 72, 1982 (1994); Phys. Rev. C 53, 2086 (1996).

[20] C.A. da Rocha and M.R. Robilotta, Phys. Rev. C 49, 1818 (1994); Phys. Rev. C 52, 531 (1995); M.R. Robilotta and C.A. da Rocha, Nucl. Phys. A615, 391 (1997).

[21] J.L. Ballot, et al., Phys. Rev. C 57, 1574 (1998).

[22] N. Kaiser, et al., Nucl. Phys. A625, 758 (1997); Nucl. Phys. A637, 395 (1998).

[23] N. Kaiser, Phys. Rev. C 61, 014003(1999); Phys. Rev. C 62, 024001(2000); Phys. Rev. C 64, 057001(2001); Phys. Rev. C 65, 017001(2002).

[24] E. Epelbaum et al., Nucl. Phys. A637, 107 (1998); Nucl. Phys. A671, 295 (2000).

[25] E. Epelbaum et al., Eur. Phys. J. A15, 543 (2002).

[26] E. Epelbaum et al., nucl-th/030810.

[27] D.R. Entem and R. Machleidt, Phys. Lett. B524, 93 (2002); Phys. Rev. C 66, 014002 (2002).

[28] D. R. Entem and R. Machleidt, nucl-th/0304018.

[29] C.E. Allgower et al., Phys. Rev. C 60, 054001 (1999); C.E. Allgower et al., Phys. Rev. C 60, 054002 (1999).

[30] F. Bauer et al., Phys. Rev. Lett. 90, 142301 (2003); M. Altmeier et al., Phys. Rev. Lett. 85, 1819 (2000).

[31] R. A. Arndt, I. I. Strakovsky, and R. L. Workman, Phys. Rev. C 62, 034005 (2000); see also: SAID, Scattering Analysis Interactive Dial-in program (http://gwdac.phys.gwu.edu).

[32] C. Elster et al., Phys. Rev. C 38, 1828 (1988).

[33] J. Haidenbauer et al., Phys. Rev. C 48, 2190 (1993).

[34] D. Hadjimichef, J. Haidenbauer, and G. Krein, Phys. Rev. C 66, 055214 (2002); ibid. C 63, 035204 (2001).

[35] G. Krein, contribution to this conference. 